\title{
Article \\ Air Corridors: Concept, Design, Simulation, and Rules of Engagement
}

\author{
Sabrina Islam Muna ${ }^{1}$,Srijita Mukherjee ${ }^{2}$, Kamesh Namuduri ${ }^{1}$ (D)0000-0003-1051-9925, Marc Compere ${ }^{3}$, M. Ilhan \\ Akbas $^{3}$, Péter Molnár ${ }^{4}$,Ravichandran Subramanian ${ }^{5}$
}

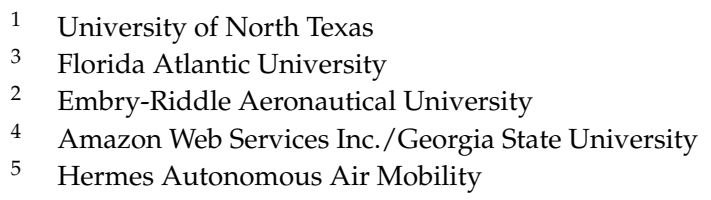

\begin{abstract}
Air corridors are an integral part of the advanced air mobility infrastructure. They are the virtual highways in the sky for transportation of people and cargo in the controlled airspace at an altitude of around $1000 \mathrm{ft}$. to $2000 \mathrm{ft}$. above ground level. This paper presents fundamental insights into the design of air corridors with high operational efficiency as well as zero collisions. It begins with the definitions of air cube, skylane or track, intersection, vertiport, gate, and air corridor. Then a multi-layered air corridor model is proposed. Traffic at intersections is analyzed in detail with examples of vehicles turning in different directions. The concept of capacity of an air corridor is introduced along with the nature of distribution of locations of vehicles in the air corridor and collision probability inside the corridor are discussed. Finally, results of traffic flow simulations are presented.
\end{abstract}

Keywords: Air Corridors, Unmanned Air Vehicle, Vehicle-to-Vehicle Communications, Geofence, Capacity, Collision-Avoidance

\section{Introduction}

Air corridors are three-dimensional (3D) volumes of airspace reserved for Unmanned Aircraft Systems ${ }^{1}$ (UASs) for Advanced Air Mobility (AAM) traffic [1,2]. Air corridor design specifications are specific to each country and are defined by its respective federal aviation authority. In the United States, the Federal Aviation Administration (FAA) defines air corridors in class B, C, or D airspace [3]. The definition of an air corridor is flexible in nature. FAA has the right to open or close an air corridor. The FAA also defines the expected performance requirements of a UAS flying in an air corridor. The design of air corridors, traffic rules in air corridors, safety requirements, and performance specifications are still evolving. Airspace design concepts, such as geofences [4], are currently being considered by various research groups.

Citation: Lastname, F.; Lastname, F. Lastname, F. Title. Preprints 2021, 1, 0 . https://doi.org/

Received:

Accepted:

Published:

Publisher's Note: MDPI stays neutral with regard to jurisdictional claims in published maps and institutional affiliations.

\subsection{Major contributions}

This paper takes a first attempt to the design of air corridors with high efficiency as well as zero collisions. It begins with the definitions of air cube, skylane or track, intersection, vertiport, gate, and air corridor. Then a multi-layered air corridor model is proposed. Traffic at intersections is analyzed in detail with examples of vehicles turning in different directions. The concept of capacity of the air corridor, location distribution of vehicles and collision probability inside the corridor are discussed. Finally, results of traffic flow simulations are presented.

\subsection{Organization}

This paper is organized as follows: Section 2 gives a summary of recent developments

1 In this paper, the terms UAS and Unmanned Aerial Vehicle (UAV) are used interchangeably for convenience. However, by definition, a UAS

\footnotetext{
includes a $U A V$, its ground control station, and a human operator.
} 
in this topic. Section 3 provides definitions and notations used in this paper. This section begins with the existing definition of geofences [4], and derives several new definitions including an air cube, skylane, intersection, vertiport, gate, and air corridor. Section 4 presents the design and traffic management method of multi-layered air corridors. This model describes how UAVs can move using skylanes and how they can make turns at some designated places called intersections. The strategies for handling traffic at intersections are discussed with examples at section 5. Section 6 presents capacity analysis of skylanes. The airspace capacity in terms of number of UAVs as well as estimated travel time is discussed here. Section 7 discusses the mobility model and the probability density function of the vehicle locations in a skyline. Section 8 derives the probability of collision in a skylane. Section 9 presents the results of simulations of slow and fast traffic flows. Section 10 concludes the paper with summary and final thoughts.

\section{Literature Review}

There are numerous applications for UAVs in military and civilian fields. Sample applications include: (1) providing communication services by serving as base stations (BSs) [5], [6], (2) monitoring air pollution or toxic gas leakage [7,8], (3) transporting cargo [9], (4) disaster prediction, assessment, and response [5], and (5) providing cost-effective wireless connectivity for devices without infrastructure coverage [10].

In the near future, (unmanned) air taxis are expected to be deployed in rural and urban areas to transport people and cargo from one place to another. Air ambulances are expected to be deployed to provide first responder services. Unlike commercial flights, UAVs are highly flexible with uncertain movement patterns, which makes UAV traffic analysis more challenging. Not only regulations, but also safety and efficiency are the key concerns of UAS Traffic Management (UTM) systems. Further, UAV traffic needs to be restricted from flying above private properties, parks, highways, and other heavily populated areas. As a result, pre-defined flight plans, and deterministic flight trajectories are needed because of the limitation of airspace. In [11] the authors describe how less structured airspaces with free flight movement allow for greater capacity and route efficiency. They also point out that free flights require greater technological capabilities and compromise safety. On the other hand, more restrictive structures, such as skylanes, enable the operations of less-equipped aircraft at the expense of slightly increased delays. Designated sections of controlled airspace, which

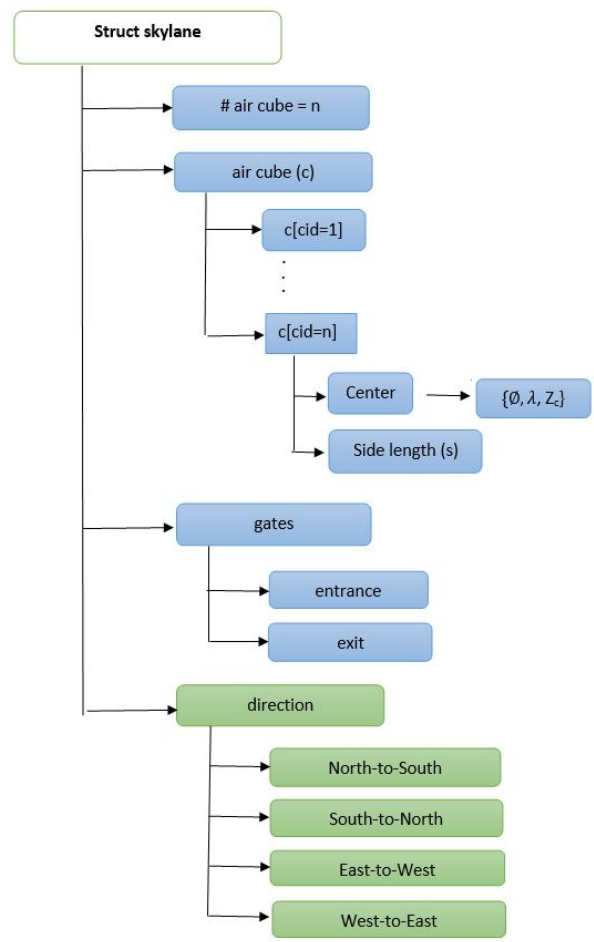

Figure 1. Skylane structure. Blue boxes represent variables that define the data elements of skylane. Green boxes represent variables that define movement directions of vehicles inside the skylane. are known as air corridors, not only improve safety and assure compliance but also enable higher tempo Urban Air Mobility (UAM) operations while trying to minimize the impact on other air traffic. Corridors could have multiple routes with different performance requirements, which provide predictability and structure, increase throughput and minimize bottlenecks. Due to the well-defined structure of an air corridor, the overheads such as communication, handovers, traffic man- 
agement for UAVs flying in an air corridor are minimal as compared to those that are flying outside the air corridor [2].

Aircraft systems operating in air corridors follow specific procedures defined for these corridors. Some specific characteristics, such as routes are static but used in a flexible fashion. FAA controls which routes are open or closed for traffic at any given time. Some design considerations for the route selection include airspace class, noise levels, departure/approach flows, final approach paths etc. Routes are charted and made available to other airspace users and periodically redefined as operational demand changes [12].

\section{Definitions and Notations}

Airspace is organized in terms of its building blocks - air cubes, skylanes, geofences, intersections and air corridors. Mathematical definitions of these building blocks will be discussed in this section. UASs also require vertiports for takeoff and landing operations.

Definition 3.1 (Geofence). [4] In the context of UAS, the term geofencing is used to describe virtual three dimensional "boundaries" each UAS flies within or avoids as a no-fly zone (NFZ).

$$
g=\left\{n, v[], z_{f}, z_{c}, m, h[], i d s[]\right\}
$$

A geofence $(g)$ is a volume defined by a minimum floor altitude $z_{f}$, maximum ceiling altitude $z_{c}$, and a list of $n$ horizontal vertices $v=\left[\left(x_{1}, y_{1}\right),\left(x_{2}, y_{2}\right), \ldots .,\left(x_{n}, y_{n}\right)\right]$ where $n \geq 3$. The volume is defined relative to the set of home locations, $h_{i}=\left(\phi_{i}, \lambda_{i}, z_{i}, t_{i}\right)$ where $h[]$ is a list of length $m \geq 2$. The pair $\left(\phi_{i}, \lambda_{i}\right)$ represent the respective latitude and longitude of the home location. The altitude of the home location above mean sea level (MSL) is represented by $z_{i}$ and the activation time for home location $i$ for $1 \leq i<m$ is represented by $t_{i}$. The variable $t_{m}$ is the deactivation time for geofence $g$. Permission to enter and operate within geofence $g$ is indicated by $i d s[]$, which contains a list of the unique identification numbers for all permitted UAV. Geofence boundaries are defined in meters relative to a home location as a vertical floor $z_{f}$ and ceiling $z_{c}$ and a list of vertices $v=v_{1}, \ldots, v_{n}$ for each $v_{i}=\left(x_{i}, y_{i}\right), i=1, \ldots, n$ where $\mathrm{n}$ is the number of vertices. The vertices define a closed simple polygon parallel to the horizontal plane. There is no convexity requirement on the polygon. The polygon is extruded to the vertical limits of the geofence to construct the geofence volume. The horizontal vertices and vertical limits are constant relative to a sequence of one or more home locations defined in set $h[$ ].

There are two types of geofences - static and dynamic. A static geofence is always active and represents unchanging boundaries such as international borders, property lines, buildings, utility poles and lines, and 24-hour airport final approach and initial departure corridors. On the other hand, a dynamic geofence is not necessarily always active, and the home location is not necessarily constant.

Definition 3.2 (Air Cube). An air cube (c) is a building block for a skylane in 3D airspace. An air cube is a static geofence in its most simplified form. All air cubes are similar in size. An air cube is exclusively reserved space for a UAV in transit at any given time. According to Near Mid-Air-Collision (NMAC) avoidance rules, the standard safe distance between two manned aircraft is $500 \mathrm{ft}$. or 152.4 meters [13]. In our model, the side length (s) of each air cube is set to 200 meters.

$$
c[c i d]=\{\text { cid,center }, \text { s, direction }\}
$$

Each air cube has a unique identifier cid which helps UAVs to understand the cube occupancy information while moving. center $=\left\{\phi, \lambda, z_{c}\right\}$, indicates the center point of the air cube. $\phi, \lambda, z_{c}$ represent latitude, longitude and ceiling altitude respectively. From the the side length (s) and the center of the air cube, the overall air cube volume can be determined. Direction indicates the traffic flow direction inside the cube. 
Definition 3.3 (Skylane or Track). A skylane (S) is a designated region of airspace for UAV (Unmanned Air Vehicle) in transit. An aircraft must fly within the skylane during its transit. A skylane can be defined as a volume consisting of a number of cubes with the same direction. An entrance gate is used to enter the skylane and one exit gate is used to exit the skylane. Gates are defined in 3.6.

$$
S[\text { sid }]=\{\text { sid, direction, } n c, c[\text { cid }], \text { gates }\},
$$

where sid represents the unique identifier of the skylane, direction represents one of the the four directions of travel [East to West], [West to East], [South to North] or [North to South]. This paper considers three layered air corridors in a typical urban or rural setting, each layer consisting of two skylanes. The top layer contains two one-directional skylanes: Southto-North and North-to-South. The bottom layer contains two one-directional skylanes: East-to-West and West-to-East. The middle-layer is used by UAVs to make turns. Figure 1 illustrates a data structure of a skylane. The length of the skylane, $L$ is equal to $n c \times s$, where $s$ is the side length of an air cube defined in meters and $n c$ is the number of air cubes.

Definition 3.4 (Intersection). An intersection is the junction where one skylane crosses another in the horizontal plane. In the skylane, it is the place where vehicles turn or change their direction. In order to avoid collisions, an intersection is designed to include three layers. The middle layer is used for a temporary hovering before a UAV actually makes the intended turn.

Definition 3.5 (Vertiport). A vertical airport or vertiport (V) is a place for take-off and landing for UAVs.

$$
V[v i d]=\left\{v i d, v[], z_{c}, i d s[]\right\}
$$

Each vertiport has a unique identifier vid. The volume of a vertiport is defined by its horizontal vertices $v\left[\left(x_{1}, y_{1}\right),\left(x_{2}, y_{2}\right),\left(x_{3}, y_{3}\right),\left(x_{4}, y_{4}\right)\right]$ and its maximum ceiling altitude $z_{c}$. The array ids[] is the sequence of identification numbers of the UAVs that are permitted to land or take off from the vertiport.

Definition 3.6 (Gate). A gate is a connection between a skylane and a vertiport. It regulates the takeoff and landing operations of the UAVs. Vehicles need to go through the gates to enter or exit the skylanes.

Definition 3.7 (Air corridor). An air corridor is a 3D volume of airspace reserved for UASs. It is a complete airspace structure that includes all skylanes, intersections, and gates.

\section{Air Corridor Design and Rules of Engagement}

In this section, the design of a multi-layered air corridor, traffic coordination, and the rules of engagement in air corridors are described. This section also illustrates through few examples how UAVs can safely make turns at intersections.

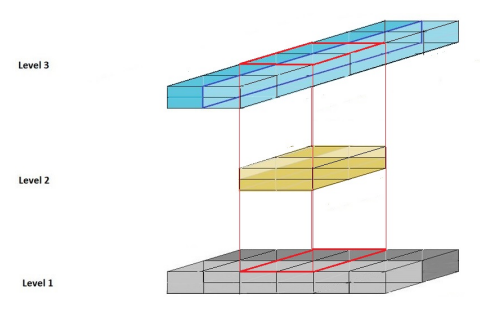

(a) Side view of an intersection

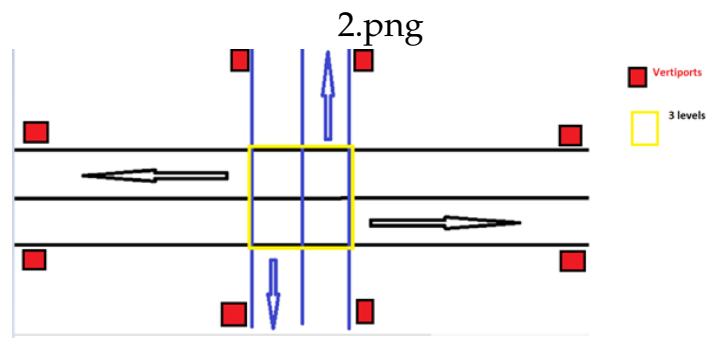

(b) Top view of an intersection: skylanes in level 1 (East-West) are in black color, skylanes in level 3 (North-South) are in blue color, and red boxes represent vertiports

Figure 2. Design of a Multi-layered Air Corridor 


\begin{tabular}{|c|l|c|l|}
\hline Symbol & Description & Symbol & Description \\
\hline$S[]$ & List of skylanes & sid & Skylane identifier \\
\hline ids[ ] & $\begin{array}{l}\text { List of identification numbers } \\
\text { of the permitted UAVs }\end{array}$ & $g c$ & $\begin{array}{l}\text { Gap between UAVs in } \\
\text { terms of number of cells }\end{array}$ \\
\hline $\mathrm{V}[]$ & Vertiport & $v i d$ & Vertiport identifier \\
\hline $\mathrm{c}[]$ & List of air cubes & cid & Air cube identifier \\
\hline $\mathrm{nc}$ & Number of air cube & $\mathrm{s}$ & Side length of air cube \\
\hline $\mathrm{g}$ & Geofence & $h_{i}$ & Home location \\
\hline $\mathrm{n}$ & Number of vertices in geofence & $v$ & list of vertices in geofence \\
\hline$z_{f}$ & Minimum floor altitude & $z_{c}$ & Maximum ceiling altitude \\
\hline$\phi$ & Latitude & $\lambda$ & Longitude \\
\hline$z_{i}$ & Altitude & $t_{i}$ & Activation time \\
\hline $\mathrm{C}$ & Capacity & $\mathrm{N}$ & Number of UAVs \\
\hline 1 & Number of skylanes & $\mathrm{L}$ & Length of a skylane \\
\hline $\mathrm{T}$ & Travel time & $T_{\text {delay }}$ & Delay time \\
\hline $\mathrm{SD}$ & Safety distance & $\alpha$ & Acceleration \\
\hline$u_{\max }$ & Maximum speed & $u_{\min }$ & Minimum speed \\
\hline$u_{i}$ & Speed of vehicle $\mathrm{i}$ & $x_{i}$ & Position of vehicle i \\
\hline
\end{tabular}

Table 1: Notation

\subsection{Multi-layered air corridor}

In the multi-layered air corridor model, the airspace is divided into two layers throughout the airspace except for intersections (Fig. 2). The top layer accommodates southbound and northbound traffic whereas the bottom layer accommodates eastbound and westbound traffic. At intersections, there is also a middle layer which is used by the vehicles for hovering when changing directions. Each layer is represented by its floor and ceiling altitudes $\left\{Z_{f}[k], Z_{c}[k]\right\}, \mathrm{k}=1,2,3$. The directions of the skylanes are fixed in top and bottom layers. As a result, the vehicles could move only in the predefined directions in these two layers. If a vehicle wants to change its direction, it needs to come to the middle layer, change its direction, and then go to the desired layer. If the air cube at the desired level is occupied, the vehicle has to wait in the middle layer briefly until the air cube becomes available. The main purpose of middle layer is to avoid collision while vehicles make their turns. This design puts the burden of collision avoidance on the vehicles. As long as the following rules of engagement are enforced, collisions can be avoided: (1) At any given time, a cube can be occupied by only one vehicle. (2) A vehicle needs to make sure that the air cube it is entering at time $(t+1)$ is going to be empty at time $(t+1)$.

\subsection{Flight path from one vertiport to another}

If a vehicle wants to travel from the south vertiport to the west vertiport, it will use the skylane from south to north at level 3, travel to an intersection, change its altitude to level 2 . If level 1 is empty at (next) time instant $(t+1)$, then the vehicle will go to level 1 switching to the westbound skylane to reach its destination. Otherwise, the vehicle will hover in the middle layer until the level 1 becomes empty. The route is indicated in green in Figure 3.

\section{Intersection Handling}



Figure 3. Flight path from south vertiport to west vertiport in multi-layered air corridor 
Intersections contain three levels to support vehicles while they change the direction of flight. When a vehicle needs to make a turn, it first goes to the middle layer (level 2), changes its heading and then goes to the desired level. Before going to the desired level, the vehicle will check the occupancy of its desired level at the next time step. If the desired level (1 or 3 ) is occupied by another UAV at that time the UAV hovers in the middle layer until the desired level is empty.

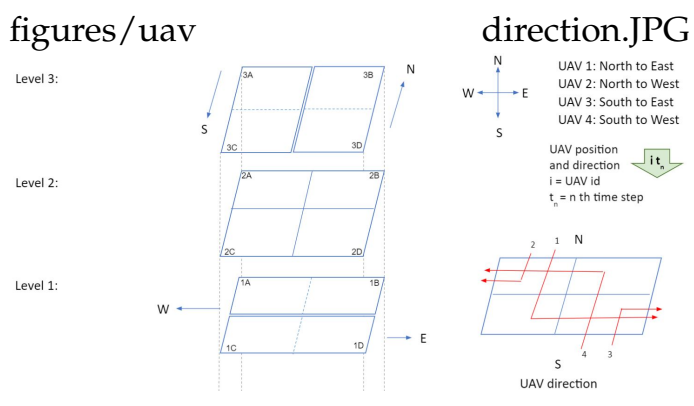

Figure 4. Designing traffic intersections in air corridors

Figure 4 illustrates a three level intersection in which each level contains four air cubes. The four cubes in level 1 are 1A, 1B (for westbound vehicles), 1C and 1D (for eastbound vehicles); cubes in level 3 are labelled as 3A, 3C (for southbound vehicles), 3B and 3D (for northbound vehicles); Cubes in level 2 are 2A, 2B, 2C, and 2D and these cubes are used for hovering. Traffic patterns at intersections are illustrated with four examples in Figure 5. Here, four UAVs are simultaneously turning at the intersection: (1) UAV 1 is turning from north to east, (2) UAV 2 is turning from north to west, (3) UAV 3 turning from south to east, and (4) UAV 4 is south to west. For simplicity, gap $(g)$ between cubes is set to zero. The traffic management at intersection is outlined in the following four steps.

1. Time step 1: At time $t_{1}$, only two vehicles are inside the intersection: UAV 1 in cube $3 \mathrm{~A}$ and UAV 4 in cube 3D (Figure 5a).

2. Time step 2: In step $2\left(t_{2}\right)$, all vehicles move one cube further. Thus, 3A and 3C are occupied by UAV 2 and 1 respectively. Similarly, UAV 3 and 4 go to cube 3D and 3B respectively (Figure 5b).

3. Time step 3: In this step, all vehicles will change their heading direction to make a turn. AT time step $3\left(t_{3}\right)$, all four UAVs will change their altitude and move to level 2 from level 3 (Figure 5c).

4. Time step 4: Two possible scenarios arise in step 4. In the first scenario, UAVs observe that all cubes in level 1 are empty. So, the UAVs will go to level 1 at time step 4 (Figure $5 \mathrm{~d})$. In the second scenario, assume that cubes 1B and 1D in level 1 are occupied by UAV 5 and UAV 6 respectively, at time step 4. So the negotiation between UAVs can be carried out in two possible ways. In the first approach (Figure 5e), both UAV 3 and 4 will stay in level 2 (in cubes $2 \mathrm{D}$ and $2 \mathrm{~B}$ ), and UAV 1 and UAV 2 will move to level 1 (to cubes $1 \mathrm{~A}$ and $1 \mathrm{C}$ ) at step 4 . UAV 4 and UAV 3 will move towards level 1 at time step 5 and time step 6 respectively. In this negotiation process, the UAV that finds an empty cube first, will get the right of way. In the second approach (Figure 5f), UAV 1, 3, 4 will stay at level 2 (in cubes 2A, 2D, and 2B) and UAV 2 will move to level 1 (1A) at time step 4. UAV 4, UAV 1, and UAV 3 will go to level 1 at time step 5 and keep moving towards their destination. In this negotiation procedure, UAVs in the front get priority over the ones that are behind. This intersection design allows implementation and utilization of multiple negotiation procedures.

\section{Capacity of an Air Corridor}

In this section, the concept of capacity of an air corridor is introduced and analyzed. The notion of capacity of an air corridor captures the idea of how many vehicles can safely fly in a given volume of airspace.

Definition 6.1 (Capacity). Capacity of an air corridor is defined by the maximum number of vehicles that can fly in the corridor maintaining minimum safe distance among them.

Consider a unit cube with 1 unit volume and assume that it is divided into smaller cubes of side length $s$. If one UAV is allowed in each smaller cube, the capacity of the unit cube is would be $\frac{1}{s^{3}}$ where $s<<1$. In the three-layered air corridor model described in Section 4 , 
the top and bottom layers are utilized for traffic and the middle layer is used for hosting the vehicles preparing for turns. In this model, the available space in the air corridor is limited to two layers. Hence the capacity of the air corridor is $\frac{2}{3 \times s^{3}}$ where $s<<1$. If one considers a skylane, the available airspace depends on the length of the skylane $(L)$, the side length of the air cube $(s)$ and the minimum gap between the UAVs in terms of number of cubes $(g c)$. Thus, the capacity $\left(C_{\text {skylane }}\right)$ of a skylane can be computed as

$$
C_{\text {skylane }}=\left\{\frac{L}{(g c+1) \times s}\right\} .
$$

For example, if the length of a skylane is $10 \mathrm{~km}$, the side length of each cube is $200 \mathrm{~m}$, and the gap between two vehicles is one cube, the capacity of the skylane is going to be 20 .

\subsection{Travel Time}

Assuming that length of a cube $s$ and there are $n c$ number of cubes in a route, the travel time for a UAV to complete the route is given by,

$$
\text { Travel Time, } T=\frac{s \cdot n c}{u(t)}+T_{\text {delay, }}
$$

where $u(t)$ represents the vehicle speed, and $T_{\text {delay }}$ takes into account delays during takeoff, landing, and waiting time during the travel. In estimating the travel times, It is convenient to consider intervals rather than fixed values for vehicle speeds and delays. For example, the speed of a vehicle $u(t) \in\left[u_{\min }, u_{\max }\right]$ and the flight delay $T_{\text {delay }} \in$ $\left[T_{\text {delay-min }}, T_{\text {delay-max }}\right]$.

\section{Mobility Model and Stationary Node Distribution}

The distribution of vehicle locations plays an important role in many computations related to traffic modeling. Estimation of vehicle location distribution requires a mobility model. Among the existing mobility models, the authors found the Manhattan Mobility Model [14] to be the most suitable for modeling traffic in air corridors.

\subsection{Manhattan Mobility Model with Safety Distance Rules}

Figure 6 illustrates the Manhattan mobility model in an air corridor with four skylanes. South-north directional vehicles are colored in blue and east-west directional vehicles are colored in green. The intersection is shown in yellow color. At a given time instant $t$, vehicle $i$ is at position $x_{i}(t)$, and the speed of vehicle $i$ is $u_{i}(t)$. The vehicles in front and back of vehicle $i$ are represented as (i+1) and (i-1) located at $x_{i+1}$ and $x_{i-1}$ with velocities $u_{i+1}$ and $u_{i-1}$ respectively. The distance between $\mathrm{i}$ and $\mathrm{i}+1$ is $\Delta x_{i}$. Similarly, $\mathrm{j}$ represents another vehicle moving from west to east and the vehicles in front and back of $j$ are $j+1$ and $\mathrm{j}-1$ respectively. The distance between $\mathrm{j}$ and $\mathrm{j}+1$ is $\Delta x_{j}$.

The Manhattan mobility model includes a minimum safety distance (SD) requirement between vehicles which is implemented in the lanes. Let $\eta$ be a random variable (RV) uniformly distributed in $[-1,1]$ which adds randomness to the vehicle speed. The speed $u_{i}$ of vehicle $i$ is also a uniform RV in the interval $\left[u_{\text {min }}, u_{\text {max }}\right]$ and $\alpha$ denotes the acceleration of vehicles.

$$
\begin{array}{r}
u_{i}(t+\Delta t)=u_{i}(t)+\eta \alpha \Delta t \\
\text { If } u_{i}(t)>u_{\text {max }}, \text { then } u_{i}(t)=u_{\text {max }} \\
\text { If } u_{i}(t)<u_{\text {min }}, \text { then } u_{i}(t)=u_{\text {min }} \\
\Delta x_{i}(t) \leq S D, \text { then } u_{i}(t)=u_{i+1}(t)-\alpha / 2
\end{array}
$$




\subsection{Probability density function of $U A V$ locations}

The probability density function of the location $(X[t])$ of UAVs, is given by,

$$
\lim _{t \rightarrow \infty} f(X[t]=c[c i d], 1 \leq c i d \leq n c)=\frac{1}{n c},
$$

where the length of each skylane represented in terms of number of air cubes is $n c$. Expressed in terms of distance, the length $(L)$ of a skylane is the product of number of cells $(n c)$ and the side length of a cube (s), i.e., $L=n c \cdot s$. From (8), it can be deduced that the probability of an air cube being occupied by a UAV is given by $\frac{N}{n c}, N \leq n c$, where $N$ is the total number of UAVs. Lemma 1 suggests that the node distribution of UAV locations remains uniform as long as there are no obstacles in the skylane.

Lemma 1. Manhattan grid model leads to uniform distribution of locations of vehicles in a skylane. Traffic may be slow or fast, but, it always flows as long as there are no obstacles in the skylane.

The proof follows the Manhattan mobility Model with SD requirement described in ( 7) and the uniform distribution of both $u(t)$ and $\eta$.

\section{Collision Probability}

Definition 8.1 (Collision Probability). Collisions occur when two or more UAVs occupy a cube at the same time.

Collision probability is the probability of one cube occupied by more than one UAV at the same time. One way to avoid any potential collision is to enforce the rule that there can be only one vehicle in a cube. This can be accomplished with the safety distance rule described in the mobility model (7). However, collision can occur for many unforeseen situations including congestion at intersections or vehicle failures. Lemma 2 estimates the probability of no collision as a function of

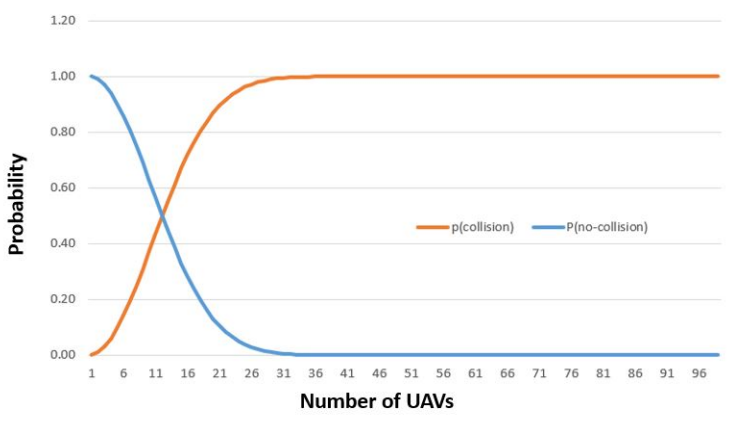

Figure 7. $P_{\text {collision }}$ and $P_{n o-\text { collision }}$ vs. Number of UAVs when $n c=100$ number of UAVs present in a skylane.

Lemma 2. Assume that there are $N$ number of $U A V$ s and nc number of cubes in a skylane and $N<<n c$. Then, the probability of no collision $\left(P_{n o-c o l l i s i o n}\right)$ is given by:

$$
P_{n o-\text { collision }}=\frac{(n c) *(n c-1) *(n c-2) * \ldots *(n c-(N-1))}{(n c)^{N}} \sim \frac{(n c)^{n c-N+1 / 2}}{e^{N} \cdot(n c-N)^{n c-N+1 / 2}}
$$

\section{Proof}

With the restriction that only one $U A V$ can be present in one cube at any given time, the number of ways $N U A V$ s can be in nc number of cubes is given by:

$$
{ }^{n c} P_{N}=\frac{n c !}{(n c-N) !} .
$$

This expression forms the numerator for the first expression on the right hand side of (9). If any UAV is allowed to occupy any cube without restriction, then the number of ways $N$ vehicles occupy $n c$ cells is given by $(n c)^{N}$. This forms the denominator for the first expression on the right hand side of (9). If both $N$ and $(N-n c)$ are large numbers, we can use Sterling's formula $\left(n ! \sim \sqrt{2 \pi n}\left(\frac{n}{e}\right)^{n}\right)$ to approximate the factorials. With this approximation, we can rewrite (11) as follows: 


$$
\begin{aligned}
& { }^{n c} P_{N}=\frac{n c !}{(n c-N) !} \sim \frac{(n c)^{n c+\frac{1}{2}}}{e^{N} \cdot(n c-N)^{n c-N+\frac{1}{2}}} \\
& \text { Probability of no collision }=\frac{\text { Number of options with restriction }}{\text { Number of options without restriction }} \\
& =\frac{{ }^{n c} P_{N}}{(n c)^{N}} \\
& =\frac{\frac{n c !}{(n c-N) !}}{(n c)^{N}} \\
& \sim \frac{(n c)^{n c-N+\frac{1}{2}}}{e^{N} \cdot(n c-N)^{n c-N+\frac{1}{2}}}
\end{aligned}
$$

Figure 7 illustrates the collision probability with $n c$ set to 100. It also suggests the best way of avoiding collisions is by enforcing the rule that there can only be one UAV in one cube.

\section{Simulations, Results, and Discussion}

This section discusses the results of discrete-time simulations carried out to demonstrate the long-term distribution of UAV locations within a skylane as a function of system parameters such as the velocity of the UAVs, traffic volume, and length of time steps. For simplicity of the simulation, the skylane is considered as a one-dimensional grid. Vehicles can move from one cube to another at a time in one direction. Since vehicles have varying speeds, some vehicles may reach the next cube between time steps and some vehicles may not. Vehicles moving at the maximum speed may advance at most one air cube per time step.

Figure 8 depicts the skylane with $n c$ cells. Traffic flows from the left to the right, starting from cube 0 . A new vehicle can enter cube 0 only when it is not occupied. Every vehicle leaves the skylane from cube $n c-1$ and presence of obstacles is not taken into account. The Manhattan mobility model allows vehicles to move forward with a given probability $\left(P_{\text {move }}\right)$ if the next cube is vacant. For a vehicle $i$ in cube $a$ at time $t$, the probabilities for the next time step $t+1$ are:

$$
P_{i, t+1}(x=a+1)= \begin{cases}0, & \text { if a }+1 \text { occupied } \\ P_{\text {move }}, & \text { otherwise }\end{cases}
$$

\subsection{Simulation comparing different velocities}

Instead of simulating variations in vehicle's velocity, the transition probabilities $\left(P_{\text {stay }}\right.$ and $\left.P_{\text {move }}\right)$ for a vehicle to advance to the next cube are defined. Higher probability of vehicle staying in the same cube $\left(P_{\text {stay }}\right)$ indicates that the traffic moves slowly. For fast moving vehicles, $P_{\text {stay }}$ in $[0.001,0.050]$ and for slow moving vehicles $P_{\text {stay }}$ in $[0.20,0.90]$. The probability of a vehicle moving to the next cube, $P_{\text {move }}=1-P_{\text {stay. }}$. The simulation was run for 100,000 time steps considering 20 vehicles in a skylane consisting of 100 air cubes.

\subsubsection{Convergence}

The standard deviation for the probability density function is much greater for slow traffic and the simulation takes much longer to converge (Figure 9).

\subsubsection{Vehicle distribution along the skylane}

The simulation results show that in the case of slow traffic the cells at the end of the skylane are less likely to be occupied (Figure 10a) even with more simulation steps (Figure 
$10 b)$.

\subsubsection{Trajectories}

Simulation results (Figure 11) show how the vehicles move along the corridor in horizontal axis. The vertical axis depicts the time. Figure 11 (b) depicts how even a single, slow vehicle impacts the traffic flow.

\subsection{Simulation comparing Different traffic volumes}

Traffic volume, i.e. ratio of vehicles to cells in skylane $\left(\frac{N}{n c}\right)$ is analyzed here. Simulation results are shown for 10 and 50 UAVs in 100 cells which correspond to the ratios of vehicles to cells 0.1 and 0.5 respectively.

\subsubsection{Probability density}

The simulation results show that the probability density along the corridor is uniform despite the variances in traffic volume.

\subsubsection{Convergence}

Simulation results show that higher traffic volume takes less time to converge.

\subsubsection{Location distribution along the skylane}

Figure 14 shows that vehicle location distribution along the skylane is going to be stationary for different traffic volumes.

\subsubsection{Trajectory}

Vehicle location distribution along the skylane is going to be uniform for different traffic volumes (Figure 15).

\section{Summary and Conclusions}

This paper provided formal definitions for air corridor and its constituent building blocks including air cubes, skylanes, intersections, vertiports, and gates. Rules of engagement for the collision-free traffic inside the corridors is discussed. Traffic management at intersections is illustrated with few examples. The notion of capacity of skylane as a function of number of cubes and gap size between UAVs is presented. Probability of collision and probability density of locations of vehicles in air corridors are discussed. Simulations of traffic in air corridors are presented to demonstrate that the distribution of vehicle locations remains uniform despite variations in traffic volumes or vehicle speeds.

\section{References}

1. NASA. Advanced Air Mobility Mission Overview. https://www.nasa.gov/aam/overview/, 2021. [Online; accessed 08-August-2021].

2. Operations Inside Corridors. https://nari.arc.nasa.gov/sites/default/files/attachments/ Operations\%20inside\%20corridors_\%20Tom\%20Prevot_FINAL.pdf, 2020.

3. Authority, F.A. Urban Air Mobility: Concept of operations; Department of Transportation, 2020.

4. Stevens, M.; Atkins, E. Geofence Definition and Deconfliction for UAS Traffic Management. IEEE Transactions on Intelligent Transportation Systems 2020, pp. 1-10.

5. Erdelj, M.; Natalizio, E.; Chowdhury, K.R.; Akyildiz, I.F. Help from the sky: Leveraging UAVs for disaster management. IEEE Pervasive Computing 2017, 16, 24-32.

6. Bor-Yaliniz, I.; Yanikomeroglu, H. The new frontier in RAN heterogeneity: Multi-tier drone-cells. IEEE Communications Magazine 2016, 54, 48-55.

7. Rossi, M.; Brunelli, D. Autonomous gas detection and mapping with unmanned aerial vehicles. IEEE Transactions on Instrumentation and Measurement 2015, 65, 765-775.

8. Akbaş, M.I.; Solmaz, G.; Turgut, D. Actor positioning based on molecular geometry in aerial sensor networks. IEEE International Conference on Communications, 2012, pp. 508-512.

9. Maza, I.; Kondak, K.; Bernard, M.; Ollero, A. Multi-UAV cooperation and control for load transportation and deployment. Selected papers from the 2nd International Symposium on UAVs, Reno, Nevada, USA June 8-10, 2009. Springer, 2009, pp. 417-449. 
10. Zeng, Y.; Zhang, R.; Lim, T.J. Wireless communications with unmanned aerial vehicles: Opportunities and challenges. IEEE Communications Magazine 2016, 54, 36-42.

11. Bauranov, A.; Rakas, J. Designing airspace for urban air mobility: A review of concepts and approaches. Progress in Aerospace Sciences 2021, 125, 100726.

12. Lacher, A. THOUGHTS ON AAM ROUTES (AKA CORRIDORS). https://nari.arc.nasa. gov/sites/default/files/attachments / AAM\%20Routes\%20-\%20NASA\%20AEWG\%20-\%20 September\%201st-Boeing-FINAL_0.pdf, 2020.

13. Johnson, S.C.; Petzen, A.; Tokotch, D. Exploration of detect-and-avoid and well-clear requirements for small UAS maneuvering in an urban environment. 17th AIAA Aviation Technology, Integration, and Operations Conference, 2017, p. 3074.

14. Fiore, M.; others. Mobility models in inter-vehicle communications literature. Politecnico di Torino 2006, 147.

Author Contributions: Conceptualization and Methodology : Sabrina Islam Muna, Kamesh Namuduri;

Simulation code: Péter Molnár;

Validation and Formal analysis: Srijita Mukherjee, Marc Compere, M. Ilhan Akbas, Péter Molnár, and Ravichandran Subramanian;

Investigation and Resources: Sabrina Islam Muna, Srijita Mukherjee, Kamesh Namuduri, Marc Compere, M. Ilhan Akbas, Péter Molnár, and Ravichandran Subramanian; data curation: Kamesh Namuduri;

Writing—original draft preparation: Sabrina Islam Muna, Kamesh Namuduri;

Writing—review and editing: Ravichandran Subramanian, Péter Molnár ;

Visualization: Sabrina Islam Muna, Péter Molnár ;

Supervision: Kamesh namuduri.

All authors have read and agreed to the published version of the manuscript.

Institutional Review Board Statement: Not applicable

Data Availability Statement: https://github.com/SabrinaIslamMuna/AirCorridor.git

Conflicts of Interest: The author(s) declared no potential conflicts of interest with respect to the research, authorship, or publication of this article.

\section{Abbreviations}

The following abbreviations are used in this manuscript:

UAV Unmanned Air Vehicle

UAS Unmanned Aircraft System

AAM Advanced Air Mobility

UAM Urban Air Mobility

UTM UAS Traffic Management

FAA Federal Aviation Administration

BS Base Station

NFZ No-Fly Zone

MSL Mean Sea Level

MAC Mid-Air-Collision 




(e) Time step 4: scenario 2, approach 1 figures/t4 s2 a2.JPG



(f) Time step 4: scenario 2, approach 2

Figure 5. Traffic management at intersections 


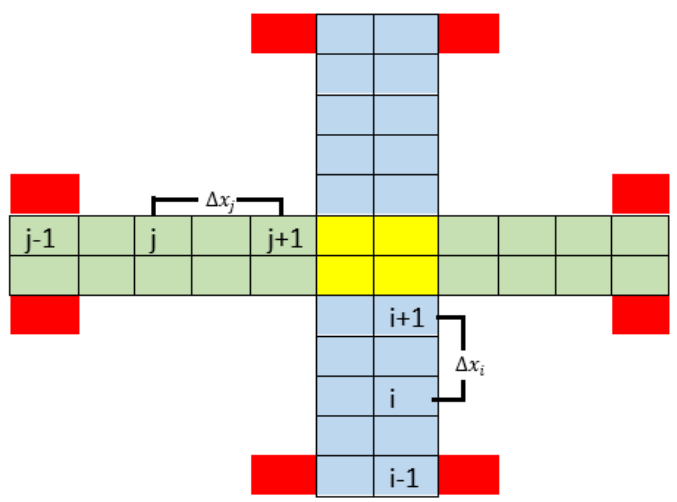

Figure 6. North-south skylanes are represented in blue color, east-West skylanes are represented in green color, intersections are shown in yellow color, and vertiports are colored in red. Indices i-1, i, and $i+1$ represent three vehicles in level three and the distance between any pair of vehicles is $\Delta x_{i}$. Similarly, $\mathrm{j}, \mathrm{j}+1$, and $\mathrm{j}-1$ represent three vehicles in level one and the distance between any pair of vehicles in this level is $\Delta x_{j}$.

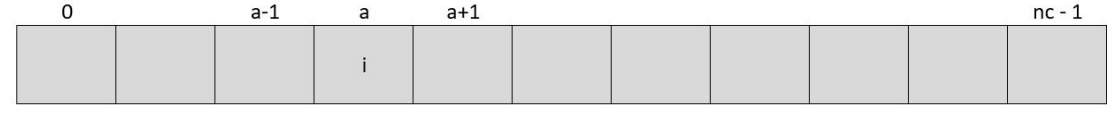

route.JPG

Figure 8. Traffic flow in a skylane with $n c$ cells

vs slow convergence.JPG
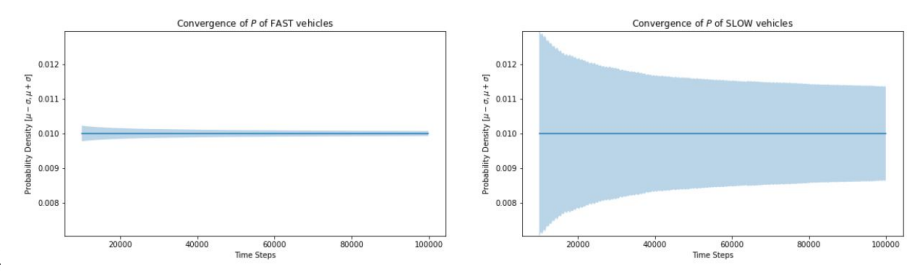

Figure 9. Convergence of FAST vs SLOW moving vehicles

vs slow distribution.JPG
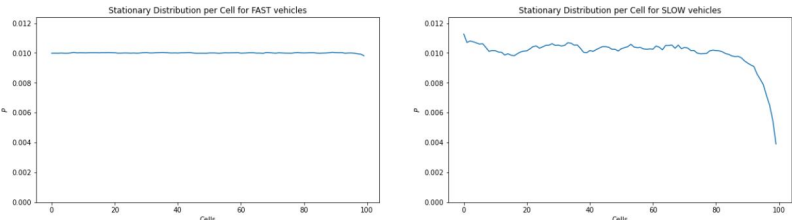

(a) Time steps $=100,000$ vs slow distribution for $100 \mathrm{t}$

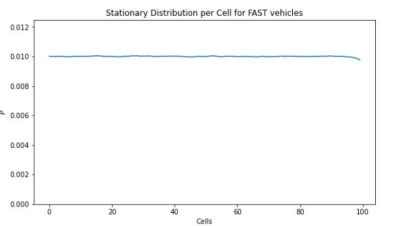

(b) Time steps $=10,000,000$

Figure 10. Location distribution of FAST vs Slow moving vehicles

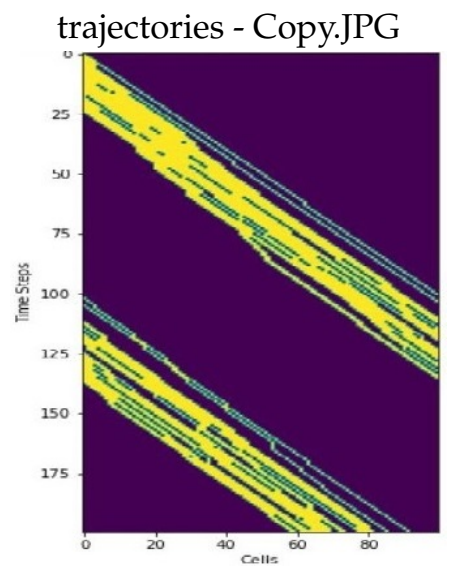

(a) FAST moving vehicles

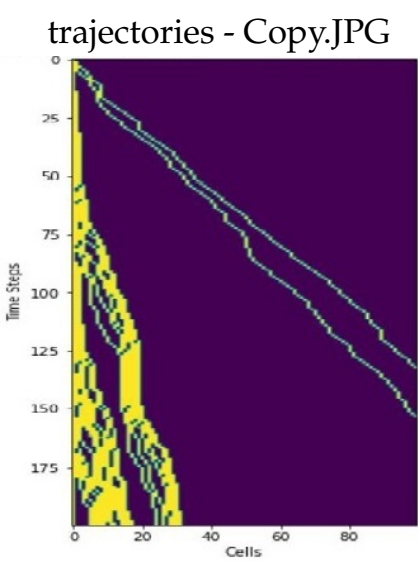

(b) SLOW moving vehicles

Figure 11. Trajectories of FAST vs SLOW moving vehicles. (Only the first 200 time steps are shown.) 


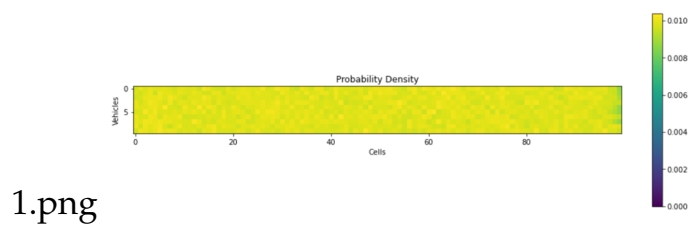

(a) Ratio of vehicles to cells in skylane $\left(\frac{N}{n c}\right)=0.1$

Figure 12. Probability density for different traffic volumes

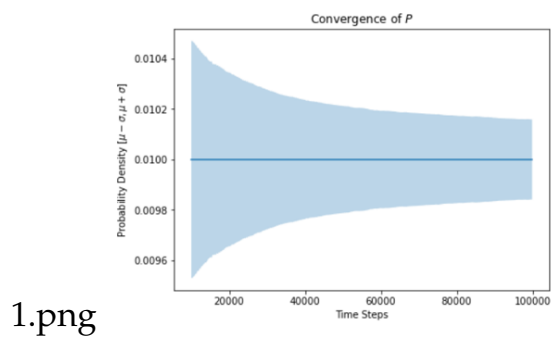

(a) Ratio of vehicles to cells in skylane $\left(\frac{N}{n c}\right)=0.1$

Figure 13. Convergence for different traffic volumes

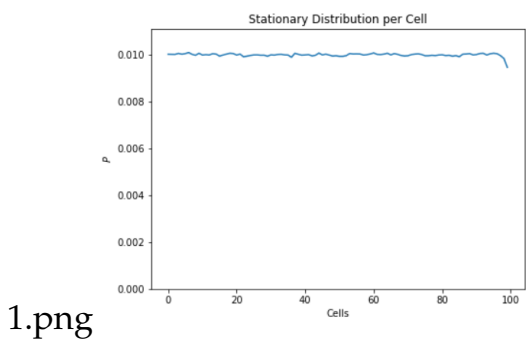

(a) Ratio of vehicles to cells in skylane $\left(\frac{\mathrm{N}}{n c}\right)=0.1$

Figure 14. Location distribution along the skylane for different traffic volumes



(a) Ratio of vehicles to cells in skylane $\left(\frac{N}{n c}\right)=0.1$

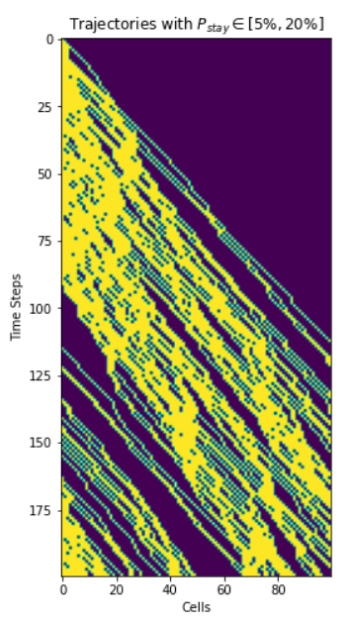
shown.) 